\section{CLINICAL AND MOLECULAR FACTORS PREDICTING STEROID RESISTANCE IN PEDIATRIC NEPHROTIC SYNDROME}

levgeniia Burlaka*, Mitiuriaeva-Korniyko Inga, Bagdasarova Ingretta. Bogomolets National Medical University

\subsection{6/archdischild-2021-europaediatrics.367}

Introduction. Nephrotic syndrome (NS) is a kidney disease characterized by albuminuria, hyperlipidemia, edema, and hypoalbuminemia. Recent data shown that more than $80 \%$ of children with nephrotic syndrome respond to steroid treatment, remain steroid-sensitive during subsequent relapses, and consequently have a favorable long-term prognosis. Steroid resistance is believed to be associated with a high risk of developing chronic renal failure. Recent reports suggest different clinical, genetic and molecular markers to be accompanied with phenomenon of steroid resistance. However, molecular markers controlling apoptosis have not been studied as a predictors of steroid resistant NS (SRNS) and steroid sensitive NS (SSNS).

Aim of the study. To identify clinical and molecular markers of the steroid-resistance phenomenon in children with NS.

Methods We analyzed 56 clinical cases of children hospitalized in Pediatric Hospital №7 (Kyiv, Ukraine) with NS (26 SSNS and 30 SRNS). Clinical data (age, gender, disease duration, blood pressure), conventional laboratory markers (serum creatinine, serum urea, GFR, blood WBC, PLT), markers of apoptosis (BcL-xL, caspase-3, caspase-8, NF-kappa B) analyzed. Stepwise logistic regression models use to identify candidates with the potential to be related to have influence of steroid resistance in children with NS. Data processed using GraphPad Prism 9.0 Software for Windows (USA, San Diego, CA).

Results Stepwise logistic regression models identified arterial hypertension as a candidate among clinical characteristics $(\beta$ : $-0,3057$; SE: 0,$1487 ; 95 \%$ confidence interval [CI]: $-0,6042$ to $-0,007281, \mathrm{p}<0,05)$ as a candidate predictive of SRNS.

Among conventional clinical markers Serum creatinine (OR: 1.04; 95\% CI: 0.95-1.1), Serum urea level (OR: 1.03; 95\% CI: 0.83-1.3), WBC level (OR: 1.4; 95\% CI: 1.1-1.8) identified as candidates predictive SRNS.

In addition, logistic regression analysis identified BcL-xL (OR: $1.3 ; 95 \%$ CI: 1.1-1.5) and caspase-3 (OR: 1.5; 95\% CI: $1.2-1.9)$ as a markers controlling apoptosis and predisposing SRNS.

Conclusion Arterial hypertension, Serum creatinine level, Serum urea level, WBC count, BcL-xL and caspase-3 levels indentified as candidate biomarkers to predict SRNS in pediatric NS.

\section{AGE-SPECIFIC EXCRETION OF CALCIUM, OXALATE, CITRATE, AND GLYCOSAMINOGLYCANS AND THEIR RATIOS IN HEALTHY CHILDREN AND CHILDREN WITH UROLITHIASIS}

Daniel Turudic, Anja Tea Golubic, Marko Bilic, Danko Milosevic*. University of Zagreb, School of Medicine; General Hospital Zabok and Croatian Veterans Hospital, Zabok, Croatia

10.1136/archdischild-2021-europaediatrics.368
We analyzed children with urolithiasis with age- and gendermatched healthy children as a retrospective study to ascertain any differences in urinary stone formation.

The study was conceived as a retrospective analysis of urinary stone disease in children from different parts of Croatia who had been treated for at least one urinary stone occurrence. For the urine samples to reflect a natural nutrient and fluid intake, the children were on a free diet. For the measurement of $\mathrm{Ca}$, Ox, Cit, and creatinine, 24-hour urine collection was performed three days in a row. We used 24-h samples instead of 2-h morning or 12-h urine samples to acquire $\mathrm{Ca}$, Ox, Cit, GAGs, and creatinine excreted in urine as accurately as possible. Urine excretion of $\mathrm{Ca}(\mathrm{mmol} / \mathrm{mmol}$ creatinine), Ox ( $\mathrm{mmol} / \mathrm{mol} \mathrm{cr})$, Cit $(\mathrm{mmol} / \mathrm{mol} \mathrm{cr})$, GAGs $(\mathrm{mg} /$ $\mathrm{mmol} \mathrm{cr}$ ), $\mathrm{Ca} / \mathrm{Cit}$ (mol/mmol), Ox/GAGs (mmol/g), Ox/Cit $(\mathrm{mmol} / \mathrm{mmol}), \mathrm{Ox} /(\mathrm{Cit} \times \mathrm{GAGs})(\mathrm{mol} \mathrm{Ox} \times \mathrm{mol} \mathrm{cr}) /(\mathrm{mol} \mathrm{Cit} \times$ g GAGs), and Cit/GAGs (mmol/g) were analyzed. Data analysis was performed by using Statistica for Windows version 8 and GraphPad Prism version 5. Additionally, J48 classifier was used to construct classification model for discrimination between subgroups Calcium ( $\mathrm{mmol} / \mathrm{mmol}$ creatinine) and the calcium/citrate ratio $(\mathrm{mol} / \mathrm{mmol})$ are the only variables that differentiate children before puberty from healthy children (ROC analysis confirmed only calcium/citrate as a significant variable with cut-off value $>0.84$ ). Pubertal/postpubertal children are distinguished from age- and gender-matched healthy children by the following variables: citrate $(\mathrm{mmol} / \mathrm{mol}$ creatinine), calcium/citrate ( $\mathrm{mol} / \mathrm{mmol})$, oxalate/glycosaminoglycans $(\mathrm{mmol} / \mathrm{g})$, oxalate/citrate ratios $(\mathrm{mmol} / \mathrm{mmol})$ and oxalate/(citrate $\times$ glycosaminoglycans) (mol oxalate $\times$ mol creatinine $) /(\mathrm{mol}$ citrate $\times$ g glycosaminoglycans), all were confirmed by ROC analysis with cut-off values $\leq 327.87,>1.02,>11.24,>0.12,>0.03$, respectively.

These results indicate a different risk of urinary stones development before puberty vs. pubertal/postpubertal children and increasing importance (deficiency) of citrate and glycosaminoglycans in such children. J48 classifier confirmed the importance of the oxalate/(citrate $\times$ glycosaminoglycans) and the calcium/citrate ratios with the practically applicable classification tree for distinguishing between pubertal/postpubertal children with urolithiasis with age- and gender-matched healthy children.

\section{A SUCCESSFUL RESOLUTION OF NUTCRACKER SYNDROME WITH 3D PRINTED PEEK EXTRAVASCULAR STENT IN AN ADOLESCENT BOY}

Da-li He, Heng-en Wang, Yong Jiao, Bin Wu, Dong Cui, Yan-yan Li, Yong Yang, Tiesheng Cao, Vinko Vidjak, Danko Milosevic*, Bo Zhang. University of Zagreb School of Medicine; General Hospital Zabok and Croatian Veterans Hospital, Zabok, Croatia

10.1136/archdischild-2021-europaediatrics.369

Three-dimensional printed PEEK extravascular stent was applied to treat a 14-year-old boy with nutcracker syndrome. Digital subtraction angiography revealed a segment of the left renal vein with reduced contrast filling immediately before its inflow into inferior vena cava and high-pressure gradient. The three-dimensional reconstruction model demonstrated that the LRV and the duodenum were contracted at the aortomesenteric angle, resulting in left renal vein compression from the 
abnormal high-level duodenal compartment. When duodenum coarses between the abdominal aorta and superior mesenteric artery (duodenal interposition), the left renal vein entrapment occurs even at $<90$ aortomesenteric degrees.

Three-dimensional PEEK printed extravascular stent was chosen to elevate the superior mesenteric artery and lower the duodenum position, thus relieving left renal vein compression. This extravascular application has significant advantages over open surgery, endovascular stenting and artificial vessel procedures with expanded polytetrafluoroethylene. It provides better cellular vitality by ensuring soft tissue proliferation. By reducing external acceleration and centrifugal force, a three-dimensional printed PEEK extravascular stent reduces adverse side effects. Such a stent has a distinctive personalized design, good stiffness, and durability that allows blood vessel growth, preventing stent migration and thrombosis. Therefore, it is suitable for both adult and pediatric patients. According to the abdominal ultrasound and MSCT scan, the post-operative follow-up results are satisfactory one year after surgery. The patient feels well, the blood flow in the left renal vein is not obstructed, and the blood flow velocity was average. The external stent is in place.

\section{COMPARING POINT OF CARE URINALYSIS TO LABORATORY URINALYSIS AT MATER DEI HOSPITAL}

Warren Debono*, Melise Mifsud, Amaris Spiteri. MDH

\subsection{6/archdischild-2021-europaediatrics.370}

A Study was done to analyse the reliability of point of care urinalysis done at Mater Dei Hospital Paediatric Emergency Department, which is the main hospital in Malta.

Data was collected over a 6 week period, starting from the 18th of January 2021. Reliability was assessed through comparison of the result from the point of care test to the result from the laboratory.

Furthermore if there was evidence of a Urinary Tract Infection (UTI) a urine culture and sensitivity test was performed.

Data was collected by going through the documentation of all the patients that attended the Paediatric Emergency Department during that time period.

If urine was collected for urinalysis and tested by both point of care urinalysis and official laboratory urinalysis, this was included in the data. In the presence of evidence of a UTI data regarding the culture and sensitivity was recorded. The data obtained from the laboratory was then converted to the same units as those obtained from the urinalysis done at the Paediatric Emergency Department. These were then compared and a Chi squared test was performed obtaining a $\mathrm{P}$ value for each of the subset of data collected.

Null Hypothesis - 'There is no significant difference in the values obtained from a urinalysis done at the Paediatric Emergency department and that done at the Laboratory'

$\mathrm{P}$ values obtained for each category were as follows:

Leukocytes - 0.757 Nitrites - 1 Proteins - 0.312 Erythrocytes -0.766 Ketones -0 Glucose -1

The Null hypothesis could be accepted for Leukocytes, Nitrites, Proteins, Erythrocytes and Glucose and rejected for ketones with a confidence of $\mathrm{p}=0.05$

A point of care urinalysis performed at the Paediatric emergency department is reliable to determine the presence and give a measure respectively of WCC, erythrocytes, protein and glucose. It is also reliable to detect nitrites.

The identification and quantification of ketones from a urinalysis at the Paediatric Emergency department should be backed up by a formal urinalysis performed by the laboratory.

\section{ARE THESE REALLY STONES?}

Susana Correia de Oliveira*, Francisca Dias de Freitas, Patrícia Sousa, António Maurício Fonseca, Ricardo Ramires, Cláudia Tavares. Department of Pediatrics, Hospital Senhora da Oliveira - Guimarães, Portugal

\subsection{6/archdischild-2021-europaediatrics.371}

Introduction Nephrolithiasis' recurrence is frequent and increases if an underlying metabolic abnormality is present. Although in up to $15-25 \%$ of affected children there is no risk factor identified, suspicion should be raised in the presence of exaggerated clinical manifestations with no reasonable explanation.

Case Report A eleven-year old uncircumcised adolescent male, with a family history of IgA Nephropathy, was referred to a pediatric nephrology consultation after being seen multiple times in the emergency department with chief complaint of recurrent episodes of dysuria with emission of up to seventeen $3-4 \mathrm{~mm}$ black calculus. He had no lumbar or abdominal pain, nor gross hematuria. On physical examination, Murphy's sign was negative bilaterally and rectal examination was normal. His laboratory evaluation, urinalysis and ultrasound were unremarkable and did not reveal any morphological or functional alterations. A 24-hour urine collection was performed with an elevated concentration of urinary urea but no other noteworthy alterations.

Homocysteine, methionine and homogentisic acid dosing were within normal ranges. Multiple laboratory evaluations of the stones weren't able to determine its composition. No metabolic or infectious cause for a calculus was identified.

Due to the recurrence of this situation he repeated both 24-hour urine collection and ultrasound with no significant results. The calculus was eventually sent to an outside laboratory and rubber elastomer was detected.

Careful examination of the patient's past medical history revealed he had already been evaluated in a Pedopsychiatric consultation before. After determining the stones were selfinduced, the adolescent was once more referred to Pedopsychiatry appointment. No further signs or symptoms of nephrolithiasis were reported.

Discussion Although Factitious Diseases (FD) caused by proxy have been increasingly recognized, there is a significant less awareness that children and adolescents can intentionally falsify symptoms. Even though urinary tract involvement is rare, any unexplained medical condition warrants the exclusion of self-induced FD.

\section{EVOLUTION OF EPIDEMIOLOGICAL AND BACTERIOLOGICAL PROFILES OF FEBRILE URINARY TRACT INFECTIONS IN CHILDREN}

Rania Ben Rabeh*, Sofiène Atiattalah, Salem Yahyaoui, Nada Missaoui, Msaddek Assidi, Sonia Mazigh, Samir Boukthir. Béchir Hamza Children's Hospital

10.1136/archdischild-2021-europaediatrics.372 